\title{
Nonlinear Multimode Fiber Optics
}

\author{
S. Wabnitz ${ }^{1}$, K. Krupa ${ }^{1}$, D. Modotto ${ }^{1}$,A. Tonello ${ }^{2}$, A. Barthélémy ${ }^{2}$, \\ V. Couderc ${ }^{2}$, G. Millot ${ }^{3}$ \\ ${ }^{I}$ Dipartimento di Ingegneria dell'Informazione, Università di Brescia, and Istituto Nazionale di Ottica del Consiglio \\ Nazionale delle Ricerche (INO-CNR), via Branze 38, 25123 Brescia, Italy \\ ${ }^{2}$ Université de Limoges, XLIM, UMR CNRS 7252, 123 Av. A. Thomas, 87060 Limoges, France \\ ${ }^{3}$ Université de Bourgogne, ICB, UMR CNRS 6303, 9 Av. A. Savary, 21078 Dijon, France \\ stefan.wabnitz@unibs.it
}

\begin{abstract}
We overview recent advances in the nonlinear optics of multimode optical fibers, including ultrabroadband sideband and supercontinuum generation, Kerr and Raman beam cleanup, modal modulation instabilities, four wave mixing, and second harmonic beam cleaning. (C) 2018 The Author(s)
\end{abstract}

OCIS codes: (060.4370) Nonlinear optics, fibers; (060.5530) Pulse propagation and temporal solitons; (060.2320) Fiber optics amplifiers and oscillators.

Since the observation of multimode optical solitons in a graded index (GRIN) multimode fiber (MMF) by Renninger and Wise in 2012 [1], there has been a resurgence of research activities involving optical pulse propagation and frequency conversion dynamics in MMFs. The peculiar property of these waveguides is the coupling among the temporal and spatial degrees of freedom of propagating beams, which can be controlled by properly adjusting the input conditions [2]. A spectacular manifestation of the spatiotemporal coupling process is the generation of ultrawideband sideband series resulting from the parametric resonance of oscillating multimode solitons $[2,3]$ or continuous waves $[4,5]$ in the anomalous or normal dispersion regime, respectively.

The Kerr effect leads to a self-cleaning of the transverse irregular intensity distribution resulting from the interference of multimode beams towards a well-defined and environmentally robust bell shaped beam [6,7]. The mode mixing leading to the spatial cleanup effect is associated with a complex temporal reshaping of the output multimode pulse profiles [8]. Although the Kerr beam cleaning is observed before a substantial self-phase modulation induced spectral broadening of the input pulses occurs, it can be preserved also in the presence of supercontinuum generation, whereby the multimode beam is carried by a bell-shaped spatial beam profile across its whole wavelength range $[9,10]$. Quite remarkably, Kerr beam cleaning is also obtained in a quasi-step index ytterbium doped MMF, both in the absence and in the presence of a pump laser. However the active configuration leads to substantially reduced input and path-average power thresholds [11]. Kerr beam cleaning has been exploited to obtain a spatial singlemode output and frequency conversion from a composite laser cavity including an ytterbium doped MMF [12], which may lead to the power scaling of mode-locked MMF lasers [13].

Another mechanism for achieving frequency conversion in MMF is modal four-wave mixing FWM [14]. Far detuned frequency translation has been demonstrated based on cascaded intermodal FWM in a few-mode GRIN MMF in a spontaneous [15] and seeded [16] configuration, respectively. Inter-modal modulation instability was exploited to achieve power-dependent frequency shifts from spontaneous sideband generation in a GRIN MMF [17]. Recent works have investigated the interplay of Kerr and Raman beam cleanup and supercontinuum generation in a specially conceived air-silica microstructure MMF [18]. Optical poling is known to induce a permanent second-order response in optical fibers: this effect has been exploited to demonstrate harmonic sideband generation and mutual cleaning of both fundamental and second-harmonic converted beams in GRIN MMFs [19,20].

In conclusion, nonlinear mode coupling in MMFs may be exploited for a novel generation of fiber optics devices that will enable the controllable generation and manipulation of high energy optical beams for a variety of applications, ranging from mode-locked and supercontinuum laser sources, stable beam delivery in the workplace for industrial cutting and welding, and biomedical imaging.

We acknowledge the financial support of: Horiba Medical and BPI france within the Dat@ diag project; iXcore research foundation; French National Research Agency ANR Labex ACTION; the European Research Council (ERC) under the European Union's Horizon 2020 research and innovation programme (grant agreement No. 740355); the European Union's Horizon 2020 research and innovation programme under the Marie Skłodowska-Curie grant agreement No. 2015-713694. 


\section{References}

1. W.H. Renninger, and F.W. Wise, "Optical solitons in graded-index multimode fibres," Nature Communications 4, 1719 (2012).

2. L.G. Wright, D.N. Christodoulides, and F. W. Wise, "Controllable spatiotemporal nonlinear effects in multimode fibres," Nature Photonics 9, 306-310 (2015).

3. L. G. Wright, S. Wabnitz, D. N. Christodoulides, and F. W. Wise, "Ultrabroadband Dispersive Radiation by Spatiotemporal Oscillation of Multimode Waves," Phys. Rev. Lett. 115, 223902 (2015).

4. K. Krupa, A. Tonello, A. Barthélémy, V. Couderc, B. Mohamed Shalaby, A. Bendahmane, G. Millot, and S. Wabnitz, "Observation of geometric parametric instability induced by the periodic spatial self-imaging of multimode waves," Phys. Rev. Lett., 116, 183901 (2016).

5. L.G. Wright, Z. Liu, D.A. Nolan, M.-J. Li, D.N. Christodoulides, and F.W. Wise, "Self-organized instability in graded-index multimode fibres," Nature Photonics 10, 771-776 (2016).

6. K. Krupa, A. Tonello, B. M. Shalaby, M. Fabert, A. Barthélémy, G. Millot, S. Wabnitz, and V. Couderc, ”Spatial beam self-cleaning in multimode fibres," Nat. Photonics 11, 237-241 (2017).

7. Z. Liu, L.G. Wright, D.N. Christodoulides, and F.W. Wise, "Kerr self-cleaning of femtosecond-pulsed beams in graded-index multimode fiber," Opt. Lett. 41, 3675-3678 (2016).

8. K. Krupa, A. Tonello, V. Couderc, A. Barthelemy, G. Millot, D. Modotto, and S. Wabnitz, "Spatiotemporal light beam compression from complex nonlinear mode mixing," arXiv:1711.11478 [physics.optics] (2017).

9. G. Lopez-Galmiche, Z.S. Eznaveh, M.A. Eftekhar, J.A. Lopez, L.G. Wright, F. Wise, D. Christodoulides, and R. Amezcua-Correa, "Visible supercontinuum generation in a graded index multimode fiber pumped at 1064 nm," Opt. Lett. 41, 2553-2556 (2016).

10. K. Krupa, C. Louot, V. Couderc, M. Fabert, R. Guenard, B. M. Shalaby, A. Tonello, D. Pagnoux, P. Leproux, A. Bendahmane, R. Dupiol, G. Millot, and S. Wabnitz, "Spatiotemporal characterization of supercontinuum extending from the visible to the mid-infrared in multimode graded-index optical fiber," Opt. Lett. 41, 57855788 (2016).

11. R. Guenard, K. Krupa, R. Dupiol, M. Fabert, A. Bendahmane, V. Kermene, A. Desfarges-Berthelemot, J. L. Auguste, A. Tonello, A. Barthélémy, G. Millot, S. Wabnitz, and V. Couderc, "Kerr self-cleaning of pulsed beam in an ytterbium doped multimode fiber," Optics Express 25, 4783-4792 (2017).

12. R. Guenard, K. Krupa, R. Dupiol, M. Fabert, A. Bendahmane, V. Kermene, A. Desfarges-Berthelemot, J. L. Auguste, A. Tonello, A. Barthélémy, G. Millot, S. Wabnitz, and V. Couderc, "Nonlinear beam self-cleaning in a coupled cavity composite laser based on multimode fiber," Opt. Express 25, 22219-22227 (2017).

13. L.G. Wright, D.N. Christodoulides, and F.W. Wise, "Spatiotemporal mode-locking in multimode fiber lasers," Science 358, $94-97$ (2017).

14. E. Nazemosadat, H. Pourbeyram, and A. Mafi, "Phase matching for spontaneous frequency conversion via four-wave mixing in graded index multimode optical fibers," J. Opt. Soc. Am. B 33, 144-150 (2016).

15. R. Dupiol, A. Bendahmane, K. Krupa, A. Tonello, M. Fabert, B. Kibler, T. Sylvestre, A. Barthélémy, V. Couderc, S. Wabnitz, and G. Millot, "Far-detuned cascaded intermodal four-wave mixing in a multimode fiber," Optics Letters 42, 1293-1296 (2017)

16. A. Bendahmane, K. Krupa, A. Tonello, D. Modotto, T. Sylvestre, V. Couderc, S. Wabnitz, G. Millot, "Seeded intermodal four-wave mixing in a highly multimode fiber," J. Opt. Soc. Am. B 35, 295-301 (2018).

17. R. Dupiol, A. Bendahmane, K. Krupa, J. Fatome, A. Tonello, M. Fabert, V. Couderc, S. Wabnitz, and G. Millot, "Intermodal modulational instability in graded-index multimode optical fibers," Optics Letters 42, 3419-3422 (2017).

18. R. Dupiol, K. Krupa, A. Tonello, M. Faber, D. Modotto, S. Wabnitz, G. Millot, V. Couderc, "Interplay of Kerr and Raman beam cleaning with a multimode microstructure fiber," arXiv:1801.03649 [physics.optics] (2018). arXiv:1711.11478 [physics.optics] (2018).

19. D. Ceoldo, K. Krupa, A. Tonello, V. Couderc, D. Modotto, U. Minoni, G. Millot, and S. Wabnitz, ”Second harmonic generation in multimode graded-index fibers: spatial beam cleaning and multiple harmonic sideband generation," Opt. Lett. 42, 971-974 (2017).

20. M. A. Eftekhar, Z. Sanjabi-Eznaveh, J. E. Antonio-Lopez, F. W. Wise, D. N. Christodoulides, and R. AmezcuaCorrea, "Instant and efficient second-harmonic generation and downconversion in unprepared graded-index multimode fibers," Opt. Lett. 42, 3478-3481 (2017) 